\title{
Cooperative data fusion of transmission and surface scan for improving limited-angle computed tomography reconstruction
}

\author{
Yu Liu ${ }^{\mathrm{a}, \mathrm{b}, *}$, Andreas Beyer ${ }^{\mathrm{c}}$, Philipp Schuetz ${ }^{\mathrm{a}}$, Juergen Hofmann ${ }^{\mathrm{a}}$, Alexander \\ Flisch $^{\mathrm{a}}$, Urs Sennhauser ${ }^{\mathrm{a}}$ \\ ${ }^{a}$ Empa - Swiss Federal Laboratories for Materials Science and Technology, Ueberlandstrasse \\ 129, 8600 Duebendorf, Switzerland \\ ${ }^{b}$ Swiss Federal Institute of Technology, ETH Zurich, Raemistrasse 101, 8092 Zurich, \\ Switzerland \\ ${ }^{c}$ Heidelberg University, Im Neuenheimer Feld 205, 69120 Heidelberg, Germany
}

\begin{abstract}
Limited-angle computed tomography allows faster inspection during production, but the reconstruction from limited-angle transmission data is an underdetermined problem which cannot be solved without any prior knowledge of the sample. In this paper, surface data from an optical scan is selected as prior information due to its high accuracy and availability. To incorporate this information, we have developed a new cooperative data fusion model in the compressed sensing framework. The model has been applied to numerical and experimental data and solved with a tailored algorithm. We demonstrate the benefit of the data fusion model and prove the robustness of the algorithm. The results from this study indicate that the data fusion process combines features resolved by both modalities and gives a significant increase in image quality. These improvements enable metrological measurements that are impossible with data acquired with any single modality.
\end{abstract}

Keywords: Computed tomography, Reconstruction, Compressed sensing, Limited-angle, Optical surface scan, Data fusion

\footnotetext{
${ }^{*}$ Corresponding author

Email address: Yu.Liu@empa.ch (Yu Liu)
}

Preprint submitted to NDT \& E International

May 2, 2016

(C) 2016. This manuscript version is made available under the Elsevier user license http://www.elsevier.com/open-access/userlicense/1.0/ 


\section{Introduction}

Computed tomography (CT) is an indirect imaging method to extract crosssectional information of a specimen. In an x-ray transmission CT, a volume representing the attenuation coefficients is reconstructed from a series of projection 5 images, which allows resolving material compositions of the sample under investigation and performing metrological measurements. After acquiring full angle non-truncated data along a circular trajectory using a cone beam x-ray source and a flat panel detector, the attenuation coefficients of the sample in the trajectory plan can be accurately reconstructed by filtered backprojection (FBP)

10 1, 2] up to the sampling frequency while the attenuation coefficients in the rest of the reconstruction volume can be estimated by the Feldkamp-Davis-Kress (FDK) [3] algorithm.

In many applications, a full angle scan is prohibited by the shape of the sample or the limited scanning time. The reconstruction from a partial angular range scan is referred to as limited-angle tomography. Due to the missing data, the reconstruction problem becomes underdetermined. Without prior knowledge, conventional methods fail to reconstruct the sample correctly [4].

Previous research has shown promising results by adding cooperative prior data, i.e. complementary data acquired with a different modality, such as low resolution CT scan [5] or CAD. However, using a CT image as prior data requires a repeated measurement while $\mathrm{CAD}$ of the sample is not always provided. Surface scanning data, on the other hand, can be acquired comparably easily with an optical scanner giving it a unique advantage in availability. Surface scanning refers to optical systems that measure objects through visible light and generate dense 3D polygonal meshes. Conventionally, the optical data is used as the image support during a reconstruction [6, 7]. The key requirement of conventional algorithms is a perfect, water-tight mesh surface representation and these approaches do not consider other objects in the field of view, which may lead to an over estimation of the attenuation coefficients of the sample under investigation. However, defective mesh surfaces with holes are more 
common in practice. Limitations of optical scanning arise if real-world points cannot be clearly identified in stereoscopic imaging or as part of the projected fringe pattern and as a result, the mesh contains holes. In this paper, cooperative data from surface scanning is fused with the x-ray projections during CT reconstruction.

By exploring the sparseness property of the image and making certain assumptions on the nature of the image, the solution to the limited-angle problem can be improved significantly by using compressed sensing (CS) theory. However, this is still not sufficient to lead to a unique solution to the problem, because the system matrix used in the reconstruction contains null space. Cooperative data fusion of surface data and transmission data reduces the null space and further improves the reconstruction result. In this paper, we propose a new model to fuse incomplete optical surface data with CT data. The fusing process takes place in the form of an iterative CT reconstruction with

45 prior information. Iterative reconstruction algorithms are able to incorporate noise models, complex geometry as well as more sophisticated physical models to construct a more accurate estimation of the image to be reconstructed. The optical data as prior information translates into a regularization term in this estimation.

This paper is structured as follows. In Section 2, we introduce the date fusion model to incorporate surface data with transmission CT data and utilize the alternative direction method of multiplier (ADMM) $[8$ to compute the model. In Section 3 , the improvement from the data fusion is demonstrated with simulation results and experimental results. The impact of our new data fusion model and the conclusion of our study are summarized in Section 4 .

\section{Materials and Methods}

\subsection{Model for CT imaging}

The attenuation of a three dimensional object is defined as a bounded function compactly supported on a three dimensional space, $f: \Omega \mapsto \mathbb{R}^{3}$, where 
$\Omega \subset \mathbb{R}^{3}$ is the set of coordinates in the reconstruction region. The measurement of the object is performed by projecting the object onto a $2 \mathrm{D}$ plane from a series of known angles with respect to the object [9]. The measurement process can be represented as

$$
P_{\theta} f(\mathbf{e})=\int_{0}^{L(\mathbf{e})} f(S(\theta)+n l) \mathrm{d} l
$$

where $S(\theta) \in \mathbb{R}^{3}$ is the source position with respect to the object, which depends on the angle of the measurement. $\mathbf{e}(u, v) \in \mathbb{R}^{2}$ is the coordinate of a detector pixel on a $2 \mathrm{D}$ flat panel detector, while $n$ is the normalized vector pointing from $S(\theta)$ to $\mathbf{e}$. $L(\mathbf{e})$ denotes the distance from the source to the detector element. The function $P_{\theta} f$ is referred to as a projection from angle $\theta$. The geometry is illustrated in Figure 1.

In numerical representation, the reconstruction region is discretized into $N_{x}$ 65 voxels, where $N_{x}=n_{1} n_{2} n_{3}$ and $n_{1}, n_{2}, n_{3}$ are the number of voxels in $x, y$, $z$ direction. The attenuation coefficients are represented as $\mathbf{x} \in \mathbb{R}^{N_{x}}$ with the $i^{t h}$ element of $\mathbf{x}$ being $x_{i}$. The measurement process will be approximated by replacing the integration with a summation:

$$
y_{j}^{\theta}=\sum_{i=1}^{N_{x}} A_{i j} x_{i}
$$

where $y_{j}^{\theta}$ denotes the total attenuation along the ray from the source to the center of the $j^{t h}\left(j=1, \ldots, N_{y}\right)$ detector element, where $N_{y}$ is the number of pixels on the detector. The intersecting length of this specific ray in the $i^{\text {th }}$ voxel is $\mathbf{A}_{i j}$. Placing all the attenuation values from one projection into one vector results in the representation of a forward projection

$$
\mathbf{y}^{\theta}=\mathbf{A}^{\theta} \mathbf{x}
$$

where $\mathbf{A}^{\theta} \in \mathbb{R}^{N_{x} \times N_{y}}$ is the forward projection matrix and $\mathbf{y}^{\theta} \in \mathbb{R}^{N_{y}}$ is a single projection. During a conventional cone beam CT scan, the source rotates around the object at a fixed distance while maintaining the central ray being perpendicular to the axis of rotation. To simplify the notation of a limitedangle cone beam CT model, we assume that the scanning angle is symmetric 
about the position of 0 degree, i.e. the scanning angle range is $[-\theta, \theta]$, and the $N_{\text {proj }}$ projections are uniformly distributed in the $[-\theta, \theta]$ interval. Thus, the limited-angle cone beam CT can be denoted as:

$$
\mathbf{y}^{\Phi}=\mathbf{A}^{\Phi} \mathbf{x}+\eta
$$

where $\mathbf{A}^{\Phi} \in \mathbb{R}^{N_{p r o j} \times N_{x} \times N_{y}}$ is the limited-angle cone beam CT system matrix. $\mathbf{y}^{\Phi} \in \mathbb{R}^{N_{\text {proj }} \times N_{y}}$ is the measurement result and $\eta$ models the noise occurred during the acquisition. The scan angle interval is $[-\Phi, \Phi]$. With a circular trajectory configuration, assuming the cone angle is $\theta_{c}, \Phi_{\min }=\frac{\pi}{2}+\frac{\theta_{c}}{2}$ is necessary to achieve an exact and stable reconstruction of the central plane and a good estimation in the rest of the reconstruction region, if $N_{\text {proj }}$ is sufficiently large [10]. Limited-angle CT addresses situations, where $\Phi<\Phi_{\min }$. The system matrix of limited-angle CT is underdetermined which leads to non-unique solutions. To narrow down the possible solution set, prior information is required in the reconstruction. The assumption that the image is sparse under some transformation is particularly important for industrial applications, as most of the industrial parts consist of a limited number of piece-wise constant materials. Here, we formulate the reconstruction problem in the compressed sensing framework:

$$
\mathbf{x}=\underset{\mathbf{x}}{\operatorname{argmin}}\|\psi(\mathbf{x})\|_{1}, \quad \text { s.t. } \quad \mathbf{A}^{\Phi} \mathbf{x}=\mathbf{y}^{\Phi}
$$

where $\psi$ is some sparsifying operator [5] transforming the image to sparse representations.

\subsection{Mesh processing}

The mesh generated from the surface scanning is denoted as $\mathcal{M}$, which contains holes due to the limitations of optical scanning. To transform the aligned mesh into voxel representation, we calculate the intersections between the mesh and the voxel grid [11. All the voxels that intersect with the mesh are marked as boundary voxels. This voxel grid is referred as the boundary of the object 
and is represented by a vector $\mathbf{B} \in \mathbb{R}^{N_{x}}$

$$
B_{i}=[\mathbf{B}]_{i}= \begin{cases}0, & \text { if } i \in \Omega_{B} \\ 1, & \text { if } i \notin \Omega_{B}\end{cases}
$$

where $\Omega_{B}$ contains all the indices of voxels which intersect with $\mathcal{M}$. The watertight mesh $\mathcal{M}^{\mathrm{w}}$ is fabricated by closing the holes in $\mathcal{M}$ with subdivision patches [12] and is used to generate mask $M$ for the reconstruction:

$$
M_{i}=[\mathbf{M}]_{i}= \begin{cases}1, & \text { if } i \in \Omega_{M} \\ 0, & \text { if } i \notin \Omega_{M}\end{cases}
$$

${ }_{75}$ Where $\Omega_{M}$ is the set of voxel indices that are located inside of the watertight mesh $\mathcal{M}^{\mathrm{w}}$.

\subsection{Data fusion model}

For simulation studies with numerical phantoms, the surface mesh represents the real surface of the sample without false negatives, i.e. holes. In this case, the surface mesh segments the volume into sample and air. The mask $\mathbf{M}$ is the set of all the voxels in the sample that contribute to the projection. The fusion can be implemented as an additional constraint in the reconstruction as

$$
\mathbf{x}=\underset{\mathbf{x}}{\operatorname{argmin}}\|\psi(\mathbf{x})\|_{1}, \quad \text { s.t. } \quad \mathbf{A}^{\Phi} \mathbf{D}_{\mathbf{M}} \mathbf{x}=\mathbf{y}^{\Phi}
$$

where $\mathbf{D}_{\mathbf{M}}=\operatorname{diag}\left\{M_{i}\right\}$. During each iteration, only the voxels in the mask are updated. However, the effectiveness of this method requires the mesh to be watertight and the sample to be the only object in the field of view, which, in practice, are often violated. We can close the holes by simply analyzing the curvature of the neighboring triangles if the assumption of smooth surface mesh is true and the holes are relatively small, i.e. subdivision method [12]. However, applying a patch at a hole to produce a watertight mesh is a risky operation. If there are protruding structures missing from the optical scanning, such patches will segment the image at the wrong location leaving a mask that is too tight for the object. As a result, all features outside the patch will be missing and the 
attenuation of the object will be overestimated. The surface mesh is a representation of the visible surface of the object which is an interface between air and other material. For transmission measurement, such an interface indicates that the attenuation coefficients on both sides of the mesh are different. This creates a direct link between the surface mesh and the gradient image of the object. The boundary field $\mathbf{B}$ marks the exact locations where a non-zero gradient is expected. However, the value of the gradient is unknown because the attenuation coefficients are unknown before reconstruction. The gradient of real-world images, especially the gradient images of industrial images, are sparse and a subset of non-zero elements in these gradient images are tagged in $\mathbf{B}$. If we use the gradient operator as the sparsifying operator $\psi$, we can update Equation (5), s.t. the gradients calculated at the boundaries tagged in $\mathbf{B}$ are preserved because they have a smaller weight in the cost function in Equation (9).

$$
\mathbf{x}=\underset{\mathbf{x}}{\operatorname{argmin}}\left\|\mathbf{D}_{\mathbf{B}} \psi(\mathbf{x})+\alpha \overline{\mathbf{D}_{\mathbf{B}}} \psi(\mathbf{x})\right\|_{1}, \quad \text { s.t. } \quad \mathbf{A}^{\Phi} \mathbf{x}=\mathbf{y}^{\Phi}
$$

where $\mathbf{D}_{\mathbf{B}}=\operatorname{diag}\left\{B_{i}\right\}, \overline{\mathbf{D}_{\mathbf{B}}}=\operatorname{diag}\left\{1-B_{i}\right\}$ and $\alpha \in[0,1)$.

\subsection{Implementation}

The constraint optimization problem in Equation (9) can be transformed into an unconstraint optimization problem:

$$
\hat{\mathbf{x}}=\underset{\mathbf{x}}{\operatorname{argmin}}\left\|\mathbf{A}^{\Phi} \mathbf{x}-\mathbf{y}^{\Phi}\right\|_{2}^{2}+c\left\|\mathbf{D}_{\mathbf{B}} \psi(\mathbf{x})+\alpha \overline{\mathbf{D}_{\mathbf{B}}} \psi(\mathbf{x})\right\|_{1}
$$

The parameter $c$ balances the data fidelity and the regularization. To search for the minimizer $\hat{\mathbf{x}}$, we use the ADMM method. Using a similar notation as in [13], we transform the unconstrained optimization to a constrained optimization problem with $K(\mathbf{x}, \mathbf{z})$ as the cost function:

$$
\left\{\begin{array}{l}
\text { minimize } K(\mathbf{x}, \mathbf{z})=\left\|\mathbf{A}^{\Phi} \mathbf{x}-\mathbf{y}^{\Phi}\right\|_{2}^{2}+c\left\|\mathbf{D}_{\mathbf{B}} \mathbf{z}+\alpha \overline{\mathbf{D}_{\mathbf{B}}} \mathbf{z}\right\|_{1} \\
\text { subject to } \mathbf{z}=\psi(\mathbf{x})
\end{array}\right.
$$

This optimization is solved using the Lagrange method. We introduce the Lagrange multiplier $\mu$, and relaxation variable $d$. The variables will be updated 
alternatively in each iteration:

$$
\left\{\begin{array}{l}
\mathbf{x}_{k+1}=\underset{\mathbf{x}}{\operatorname{argmin}}\left\|\mathbf{A}^{\Phi} \mathbf{x}-\mathbf{y}^{\Phi}\right\|_{2}^{2}+\mu\left\|\psi(\mathbf{x})-\mathbf{z}_{k}-\mathbf{d}_{k}\right\|_{2}^{2} \\
\mathbf{z}_{k+1}=\underset{\mathbf{z}}{\operatorname{argmin}} c\left\|\mathbf{D}_{\mathbf{B}} \mathbf{z}+\alpha \overline{\mathbf{D}_{\mathbf{B}} \mathbf{z}}\right\|_{1}+\mu\left\|\psi(\mathbf{x})-\mathbf{z}-\mathbf{d}_{k}\right\|_{2}^{2} \\
\mathbf{d}_{k+1}=\mathbf{d}_{k}-\left(\psi(\mathbf{x})-\mathbf{z}_{k}\right)
\end{array}\right.
$$
tion axis. The boundary voxels are known from the numerical phantom. The full set of boundary pixels is used as a perfect representation of the surface while a subset without all protruding and concave structures is used as a simulated boundary derived from a defective optical surface measurement (Figure 2(b)). assumed to be monochromatic with an equivalent energy of $100 \mathrm{k} e \mathrm{~V}$. Simulated Poisson noise was added to the projection assuming a total of 1 million photons per detector element during the exposure. To demonstrate the improvement 


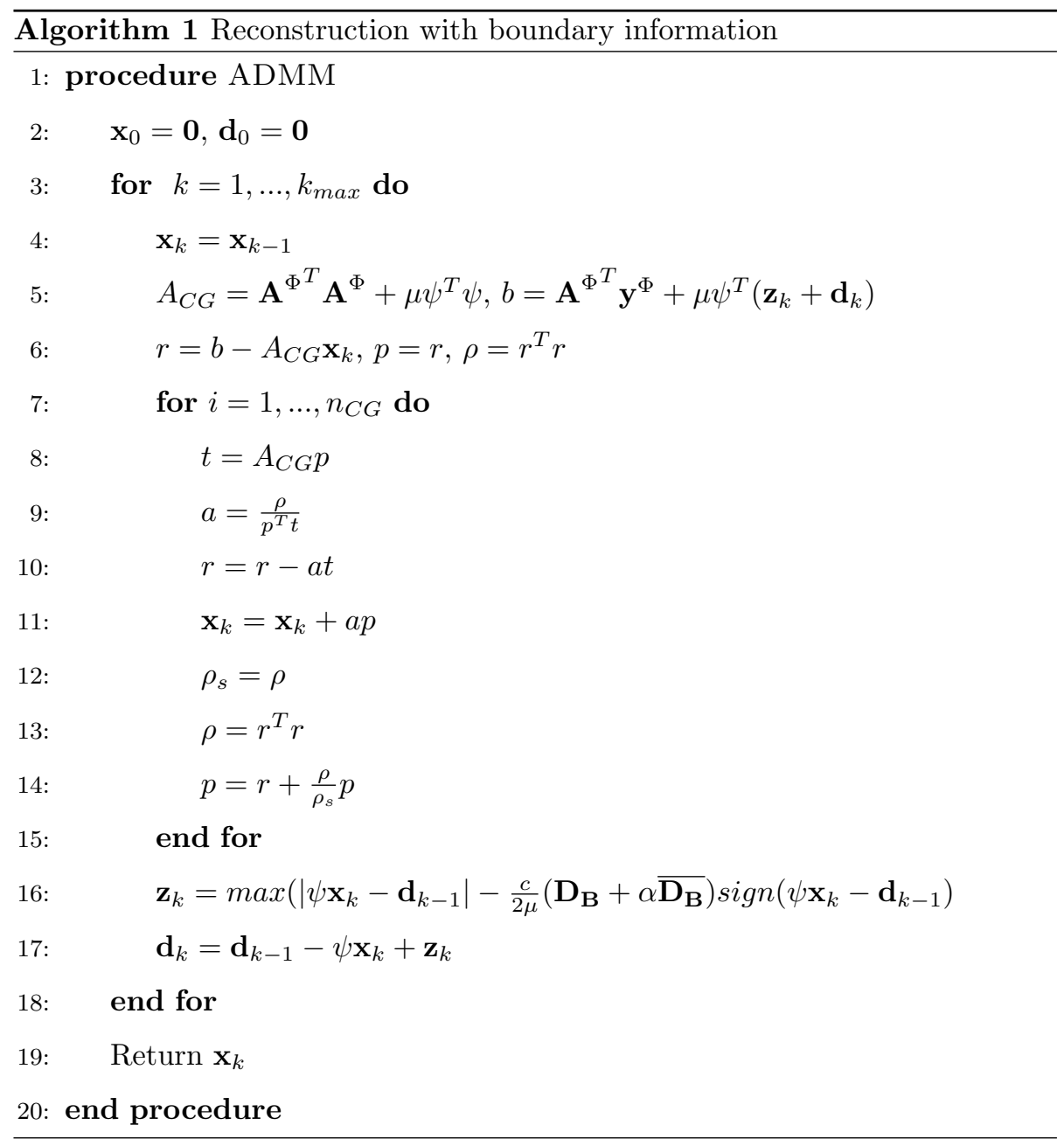


from fusing prior information with CT projection data, the same set of limited-

angle projections were reconstructed using the algorithm presented in Section 2 with complete or incomplete boundary as prior and with zero prior information.

After each iteration, we calculate the mean square error of the projections $\left(M S E_{\text {proj }}\right)$ and the reconstructed volume $\left(M S E_{\text {recon }}\right)$ as the phantom is known. In addition, the structure similarity index (SSIM) [14] of the cross-section is calculated. The SSIM is a patch-based algorithm for evaluating the image quality over the whole image. We are particularly interested in the structural component of the SSIM because correctly reconstructed geometry is essential for metrological purposes.

The combination of the parameters in the model and solver has to balance the data fidelity and the regularization strength and to guarantee the convergence of the sequence. As part of the cost function, $c$ determines the strength of the regularization term. If the value of $c$ is too small, the optical data does not have enough weight in the cost function to be significant in the result. During the solving process, $\mu$ and the ratio between $c$ and $\mu$ will also have an impact on the optimization result and speed. A poorly chosen ratio may lead to oversmoothed images. In this publication, the values are determined experimentally after a parameter sweep using combinations $a$ to $g$. The $M S E_{\text {proj }}$ is plotted against the iterations. The result is illustrated in Figure 3. Although both combination $a$ and $g$ converge to a small MSE, the combination $a$ results in a higher structural similarity, indicating a better fusion result.

In this simulation study, we set $c=1.0$ and $\mu=10$ while the number of CG iteration is set to 5 . The computation of the model was performed on a workstation with 2 Xeon ${ }^{\circledR}$ E5-2670 and 1 Tesla K20m. Each iteration takes approximately $60 \mathrm{~s}$. The $M S E_{\text {recon }}$ and the SSIM of the cross-section extracted from the reconstruction with zero prior, incomplete boundary and complete boundary are plotted in Figure $4(\mathrm{a}, \mathrm{d}, \mathrm{g})$. The $M S E_{\text {proj }}$ dropped quickly after a few iterations before it started to slightly increase due to the regularization, while the $M S E_{\text {recon }}$ decreases with the iteration. However, the reconstructions were stopped after 1000 iterations before the sequence of the reconstructed volumes 
converges. As our task is to mitigate the limited-angle artifact, primarily the blurred boundaries which are not parallel to any rays during the acquisition, the structural similarity is of more interest. The structural component of the SSIM has reached its maximum at 0.9686 for zero prior, 0.9770 for incomplete boundary and 0.9891 for complete boundary after 1000 iterations which justifies the early stopping of the algorithm. The increase of SSIM is in line with the successfully reconstructed boundaries which are parallel to $z$ axis in Figure 4(b, e). While the external features of the sample benefit directly from the fusion of surface data, we are also interested in the improvement in the reconstruction of internal features. The modulation transfer function (MTF) is calculated with the help of the test structures inside of the phantom as a measure of spatial resolution. Although the MTF is non-stationary in limited-angle CT [15] and even the local stationary assumption is inaccurate, the MTF is calculated in a standard and consistent procedure across the three images for comparison purposes. The edge spread function is extracted along the $x$ and $z$ direction at the test structures marked as $f 1$ and $f 2$ in Figure $4(\mathrm{~b})$. The MTF is derived and shown in Figure 4(c, f, i). The measurement indicates that the spatial resolution along $z$ direction is higher than the resolution along $x$ direction, which is explained by the limited scanning angle. We also noticed that the local MTF inside the phantom doesn't change if boundary information is provided to the reconstruction, which is an expected result as there is no additional information regarding the internal structures provided to the reconstruction. However, other improvements on internal features are noticeable, such as the uniformity of the image and the contrast at the test structures. To describe the property, the contrast to noise ratio $(\mathrm{CNR})$

$$
C N R=\frac{\left|\mu_{f}-\mu_{b}\right|}{\sigma_{b}}
$$

where $\mu_{f}$ is the mean value of the feature, $\mu_{b}$ is the mean value of the background and $\sigma_{b}$ is the standard deviation of the background, is calculated at the test structures. The results are listed in Table 1. The CNR at the test structures is significantly improved because of the better estimation of the pixel values inside 


\begin{tabular}{cccc}
\hline Feature & Zero prior & Defective mesh & Perfect mesh \\
\hline \hline f1 & 5.72 & 33.95 & 16.97 \\
\hline f2 & 24.52 & 35.51 & 71.47 \\
\hline
\end{tabular}

Table 1: Contrast to noise ratio at $f 1$ and $f 2$ in Figure 2 after reconstruction with zero prior, defective mesh and perfect mesh

130

the object.

In the second simulation, the phantom is a cuboid placed in the center of the CT system and is aligned to the detector such that the top and the bottom face of the cuboid are parallel to the trajectory plane. This is a typical scenario where severe cone beam artifacts arise at the top and bottom faces. The cone angle of the beam in this experiment is 20 degree and the scanning angle is 360 degree, i.e. a full circle trajectory, as we only focus on the cone beam artifact in this case. A Poisson noise level, which is equivalent to 1 million photons per detector element, is added to the simulated projection data. The phantom is then reconstructed with the standard FDK algorithm (Figure 5(a)) and our proposed algorithm incorporating surface data (Figure 5(b)). The formerly blurred top and bottom faces due to cone beam artifact are restored by the fusion of surface data. Although it is not justifiable to claim an increased spatial resolution in $y$ direction, the reconstruction of the top and bottom surfaces is crucial when segmenting the object and supporting materials in experiments. Besides the reduction of cone beam artifact, the reconstruction also benefits from the iteration to gain a much more accurate and precise voxel value as illustrated in Figure 5(b).

\subsection{Experimental study}

To validate the model and simulation and to prove the feasibility in practical applications, we applied the model to experimental data and solved it using Algorithm 1 The sample used in the experiment is an aluminum cuboid with drilling holes and trenches on the surface. The sample was scanned with a micro 
CT system and a full circular trajectory. The scanning parameters are listed in Table 2.

The FDK reconstruction from the full-angle projections was segmented to extract the object surface which in turn was used for alignment with the optical scanning result. The optical scanner generated a mesh representation $\mathcal{M}$ of the sample's surface. Due to known limitations, most of the deep trenches and holes cannot be acquired, as shown in Figure 6(d). The aligned mesh was rasterized to generate the boundary voxel map $\mathbf{B}$ for the reconstruction. A subset of the projection data, containing only projections taken in a 60 degree interval with fixed angular increment, was extracted as an input to a limitedangle reconstruction. Two reconstructions were carried out using Algorithm 1 with and without surface data. Both reconstructions used the zero image as initial image and executed 500 iterations. The computation of the model was performed on a workstation with 2 Xeon ${ }^{\circledR}$ E5-2670 and 1 Tesla K20m. Each iteration takes approximately $120 \mathrm{~s}$. The results are shown in Figure 6. The cross-sectional images of the reconstructed volumes along different directions are plotted into three groups for comparison. Figure 6 (a) and (b) show the cross section with normal direction along the central beam. The most noticeable improvement is the reduction of cone beam artifacts at the top and bottom of the image, which is important to separate the sample and other objects, such as the supporting stage. Limited-angle artifact is not prominent in Figure 6(a, b). On the contrary, the cross sections along the other two directions Figure 175 6(c, e) are distorted by the limited-angle artifact. As a result, all surfaces with normal direction along the beam direction are blurred. Due to the lack of boundary information, the reconstruction algorithm cannot stop the smearing of the voxel value across the object boundary, which is an empirical explanation of the blurring. After fusing the optical data and CT projection using Algorithm 1, the information acquired by both techniques is successfully combined. Features that were previously only available in one dataset are linked allowing more metrological tasks of the object. Unlike the simulation studies, a distortion-free and noise-free phantom is not available, rendering the full-reference metrics, 


\begin{tabular}{r|l}
\hline Parameter & Value \\
\hline \hline Source detector distance & $1020.2[\mathrm{~mm}]$ \\
Source object distance & $235.8[\mathrm{~mm}]$ \\
Source voltage & $160.0[\mathrm{kV}]$ \\
Source current & $60.0[\mu \mathrm{A}]$ \\
Source target material & Tungsten $(\mathrm{W})$ \\
Scintillation material & CsI \\
Exposure time & $1.0[\mathrm{~s}]$ \\
Averaging & $3($ frames $)$ \\
Binning factor & $2 \mathrm{x}$ \\
Angular step & $1.0[\mathrm{deg}]$ \\
Reconstruction volume dimension & $340 \times 840 \times 500($ voxels $)$ \\
Voxel pitch size & $0.12 \times 0.12 \times 0.12[\mathrm{~mm}]$ \\
\hline
\end{tabular}

Table 2: Experiment parameters

\begin{tabular}{ccc}
\hline Feature & Zero prior & Optical scan \\
\hline \hline $\mathrm{f} 3$ & 2.42 & 25.07 \\
\hline $\mathrm{f} 4$ & 4.03 & 46.27 \\
\hline
\end{tabular}

Table 3: Contrast to noise ratio at $f 3$ and $f 4$ in Figure 4 (b) after reconstruction with zero prior and defective mesh from an optical scan.

such as SSIM and MSE, impossible to calculate. The CNR in the cross-section images are used as quantitative measures of the image quality. The feature selected in this study is a drilling hole in the object 6(b), where optical data and CT data are partially available. The results are in line with the visual inspection and listed in Table 3

\section{Discussion and Conclusions}

190

In this paper, we present a new data fusion model cooperating optical scanning data with transmission data to improve the limited-angle cone beam CT re- 
construction. In contrast to previous work, this model allows objects other than the specimen to be present in the field of view and accepts defective meshes from real-world optical scans, allowing day-to-day practical use in production. The fusion of surface information gives rise to the image quality of the reconstruction, namely the contrast to noise ratio which is important for non-destructive testing, and mitigates the cone beam artifact and limited-angle artifact. The fused volume combines features resolved by both imaging techniques allowing various metrology tasks which are not possible with any data set from a single modality, although the additional surface information will not lead to extra resolving capability of internal features. On the other hand, the proposed model and computation algorithm still have several drawbacks. Firstly, the model has multiple parameters, to which the minimizer is sensitive. A parameter study should be performed at a lower resolution to test the parameter combinations. Secondly, the optimization process needs to be accelerated to achieve a high-quality reconstruction within a shorter time. Furthermore, the algorithm assumes aligned datasets which is difficult to achieve considering the limitations of either modality and the different forms of representation of information [11. Whereas, the promising results and the demand from industry encourage further research to tackle the drawbacks and continue to increase the quality of the fusion result.

\section{Acknowledgments}

This work is funded by the Swiss National Science Foundation (SNF) under the grant 200021L_141311 within the DACH framework. Our joint project ILATO is carried out with the Interdisciplinary Center for Scientific Computing (IWR) of the Heidelberg University funded by the Deutsche Forschungsgemeinschaft (DFG) under grant BO 864/17-1. The authors would like to thank Rolf Kaufmann from Empa for his help with the CT data acquisition and Susanne Krömker, Hubert Mara from IWR for offering mathematical consultations in topology and inspiring discussions. 
口 [1] F. Noo, M. Defrise, R. Clackdoyle, H. Kudo, Image reconstruction from fan-beam projections on less than a short scan, Physics in Medicine and Biology 47 (14) (2002) 2525. doi:10.1088/0031-9155/47/14/311.

URL http://iopscience.iop.org/0031-9155/47/14/311

225 [ [2] G.-H. Chen, R. Tokalkanahalli, T. Zhuang, B. E. Nett, J. Hsieh, Develop-

1. ment and evaluation of an exact fan-beam reconstruction algorithm using

1. an equal weighting scheme via locally compensated filtered backprojec-

1. tion (LCFBP), Medical Physics 33 (2) (2006) 475-481. doi:10.1118/1. 2165416 .

URL http://scitation.aip.org/content/aapm/journal/medphys/33/ $2 / 10.1118 / 1.2165416$

[3] L. A. Feldkamp, L. C. Davis, J. W. Kress, Practical cone-beam algorithm, a Journal of the Optical Society of America A 1 (6) (1984) 612-619. doi: 10.1364/JOSAA.1.000612. URL http: //josaa.osa .org/abstract . cfm?URI=josaa-1-6-612

[4] A. G. Ramm, Inversion of limited-angle tomographic data, Computers \& Mathematics with Applications 22 (45) (1991) 101-111. doi:10.1016/0898-1221(91)90135-Q.

a URL http://www.sciencedirect.com/science/article/pii/ $089812219190135 Q$

[5] G.-H. Chen, J. Tang, S. Leng, Prior image constrained compressed sensing (PICCS): a method to accurately reconstruct dynamic CT images from highly undersampled projection data sets, Medical physics 35 (2) (2008) 660-663.

${ }_{245}$ [6] Y. Liu, P. Schuetz, A. Flisch, U. Sennhauser, Exploring the limits of limited-angle computed tomography complemented with surface data, in: Proc. of the 11th European Conference on Non-Destructive Testing, 2014. 
URL http://www.ndt.net/events/ECNDT2014/app/content/topic. php?eventID $=20 \&$ TopicID $=1728$

250

口

[8] S. Boyd, N. Parikh, E. Chu, B. Peleato, J. Eckstein, Distributed Opti255

1. of Multipliers, Found. Trends Mach. Learn. 3 (1) (2011) 1-122. doi: $10.1561 / 2200000016$.

URL http://dx.doi .org/10.1561/2200000016

q [9] X. Jia, B. Dong, Y. Lou, S. B. Jiang, GPU-based iterative cone-beam CT

[10] G. L. Zeng, G. T. Gullberg, Short-scan fan beam algorithm for noncircular detector orbits, in: Proc. SPIE, Vol. 1445, 1991, pp. 332-340. doi:10.

265

[11] A. Beyer, H. Mara, S. Krömker, ILATO Project: Fusion of Optical Surface Models and Volumetric CT Data, CoRR - Computing Research Repository abs/1404.6583. arXiv:1404.6583.

$270 \quad$ URL http://arxiv.org/abs/1404.6583

[12] P. Liepa, Filling Holes in Meshes, in: Proc. of the 2003 Eurographics/ACM SIGGRAPH Symposium on Geometry Processing, SGP '03, Eurographics Association, Aire-la-Ville, Switzerland, 2003, pp. 200-205.

URL http://dl .acm .org/citation. cfm?id=882370.882397 
[13] C. Mory, B. Zhang, V. Auvray, M. Grass, D. Schafer, F. Peyrin, S. Rit, P. Douek, L. Boussel, ECG-gated C-arm computed tomography using L1 regularization, in: Proc. of the 20th European Signal Processing Conference (EUSIPCO), 2012, pp. 2728-2732.

[14] Z. Wang, A. Bovik, H. Sheikh, E. Simoncelli, Image quality assessment: from error visibility to structural similarity, IEEE Transactions on Image Processing 13 (4) (2004) 600-612. doi:10.1109/TIP.2003.819861

[15] S. Glick, M. King, K. Knesaurek, K. Burbank, An investigation of the stationarity of the 3D modulation transfer function of SPECT, IEEE Transactions on Nuclear Science 36 (1) (1989) 973-977. doi:10.1109/23.34587. 


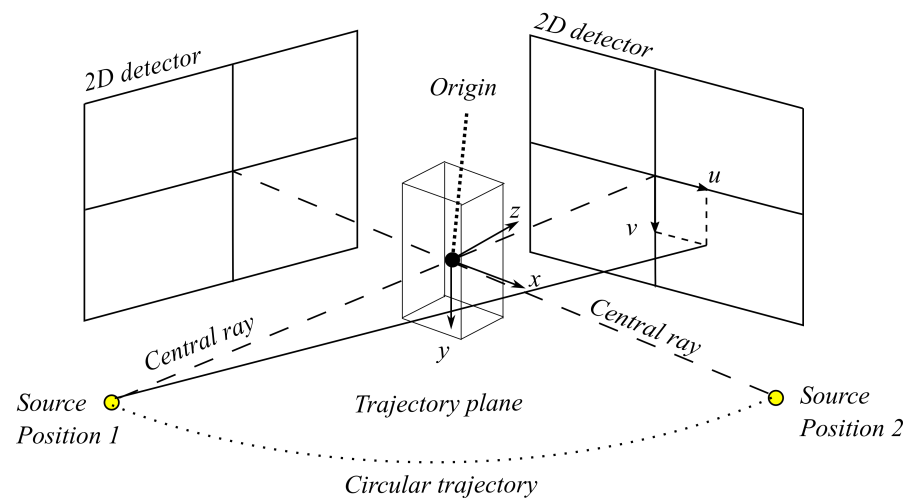

Figure 1: Schematic of the CT setup. The scanning trajectory is either a full circle in the trajectory plane or part of a circle for limited-angle scanning. The source and the detector are aligned such that the central ray from the cone beam is perpendicular to the $2 \mathrm{D}$ detector plane through the geometry center of the $2 \mathrm{D}$ detector. The distance from the source to the origin and the distance from the source to the center of the detector are fixed during the scan.

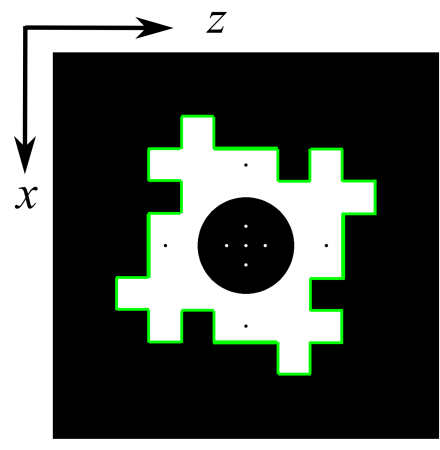

(a)

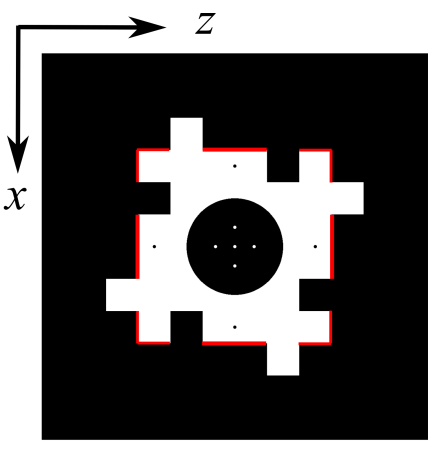

(b)

Figure 2: The cross section of the simulated phantom. The attenuation coefficients are linearly representated as gray levels. 0 is mapped to black and $0.054\left[\mathrm{~mm}^{-1}\right]$ is mapped to white, i.e. window $=[0,0.054]$. The same structure repeats in $y$ direction. In the simulation, this cross section is aligned to the trajectory plane. In (a), all the boundaries visible to the outside are highlighted in green and will be used as perfect boundary in the simulations, while in (b) only part of the boundaries are highlighted in red and they will be employed as defective boundary to simulate real-world surface scanning results with holes. 


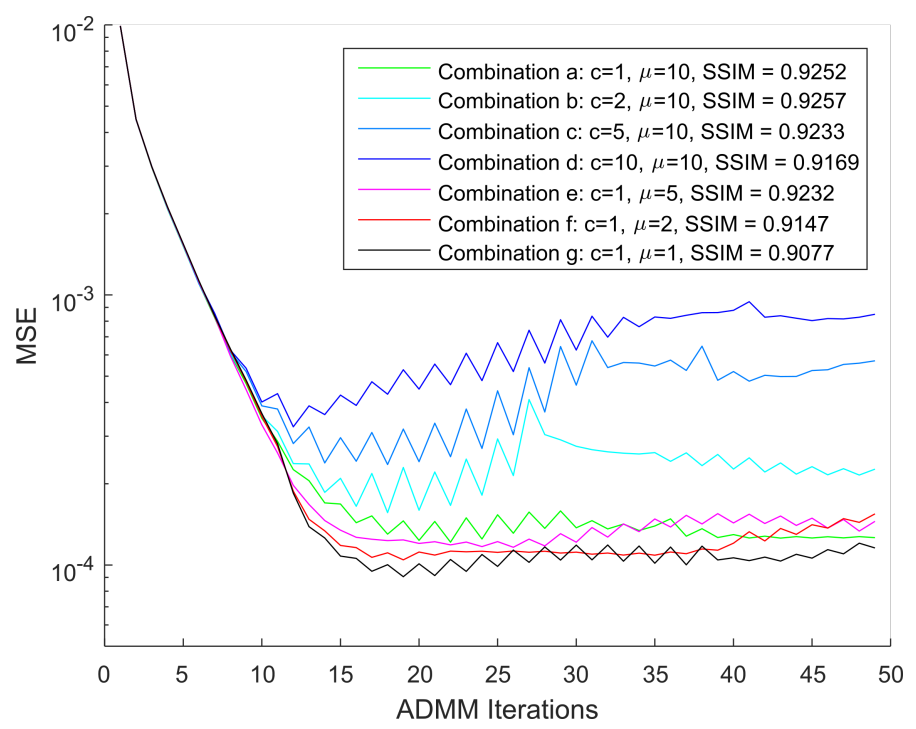

Figure 3: The MSE between the measurement and the projection of the current iteration result. The results are color coded according the combination of parameters. The structrual component of the SSIM of the last iteration result is indicated in the legend of the respective trace. 


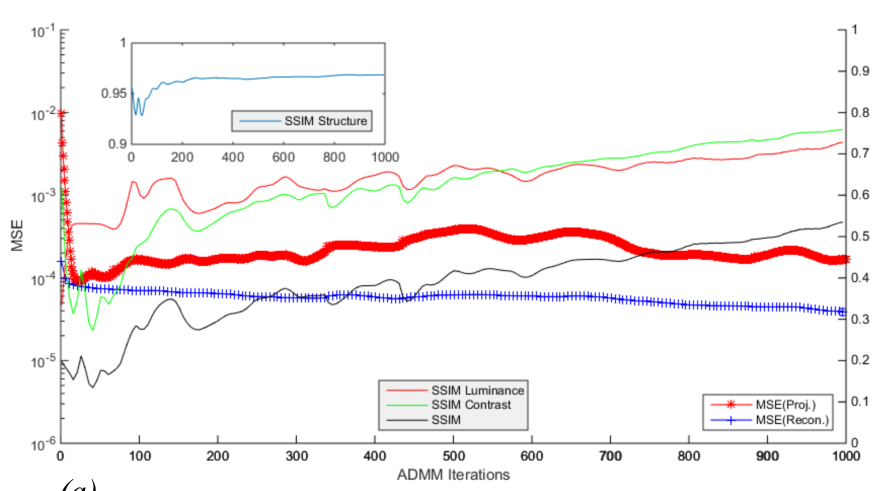

(a)

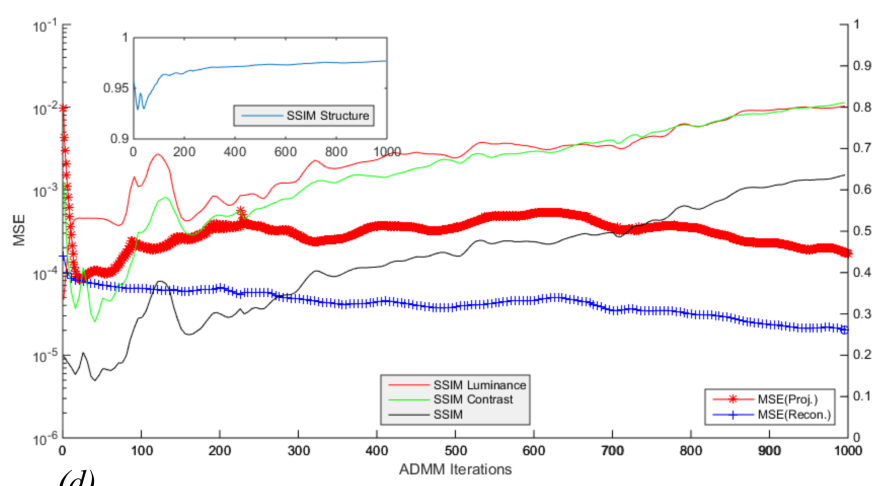

(d)

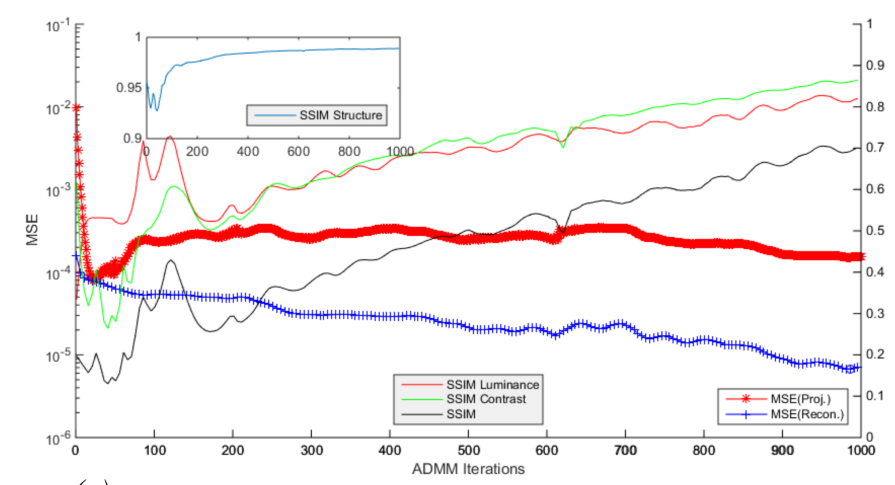

(g)

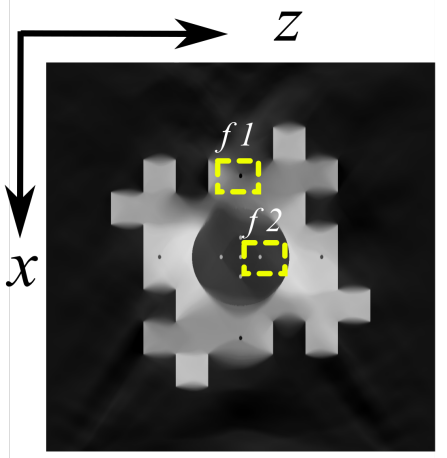

(b)

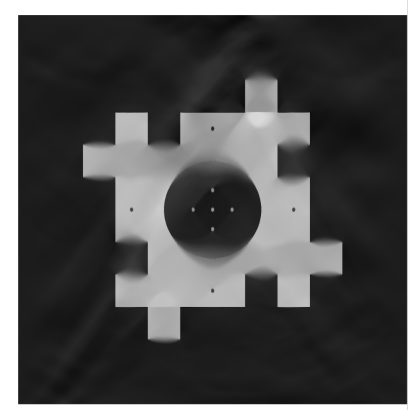

(e)
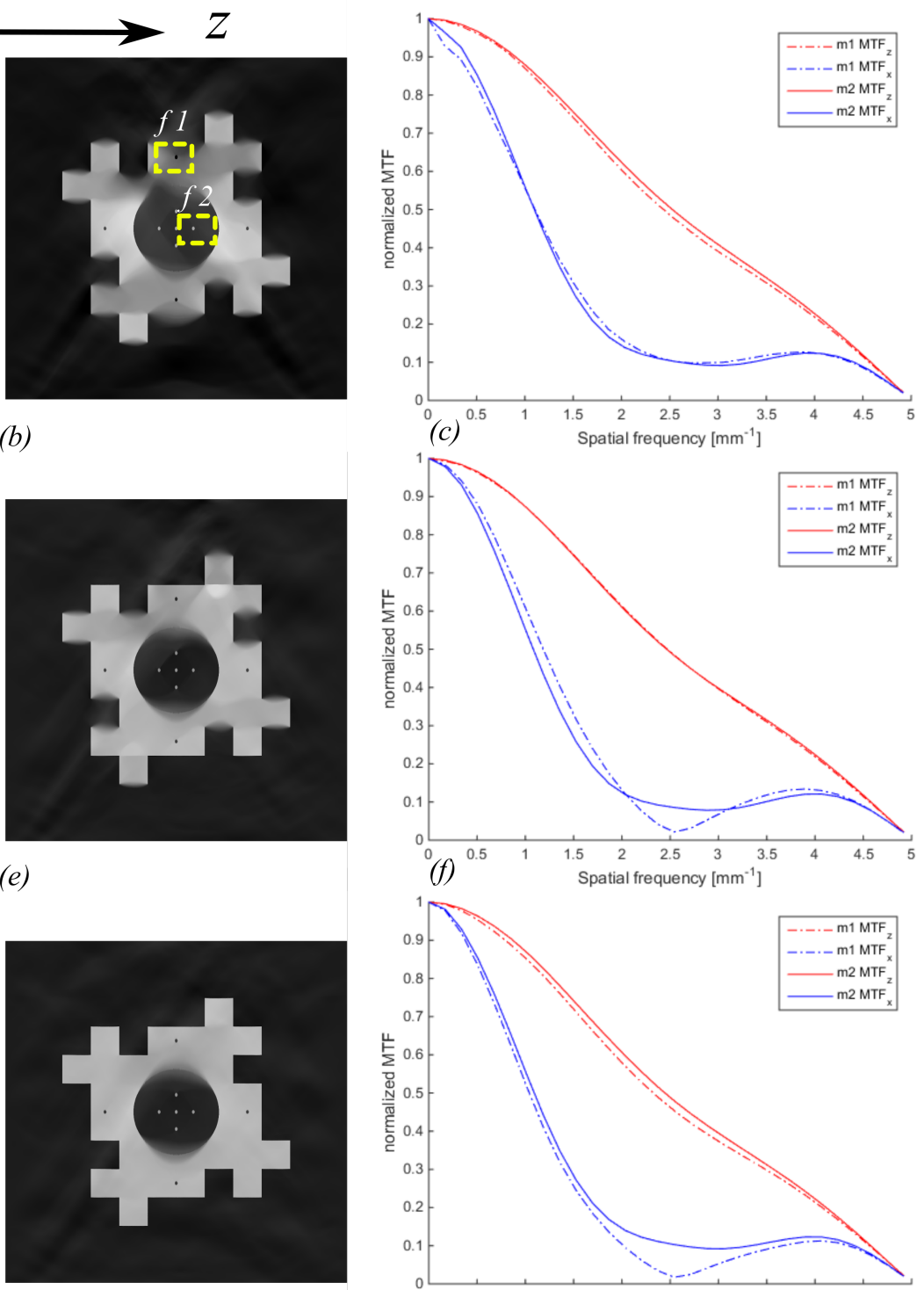

(h)

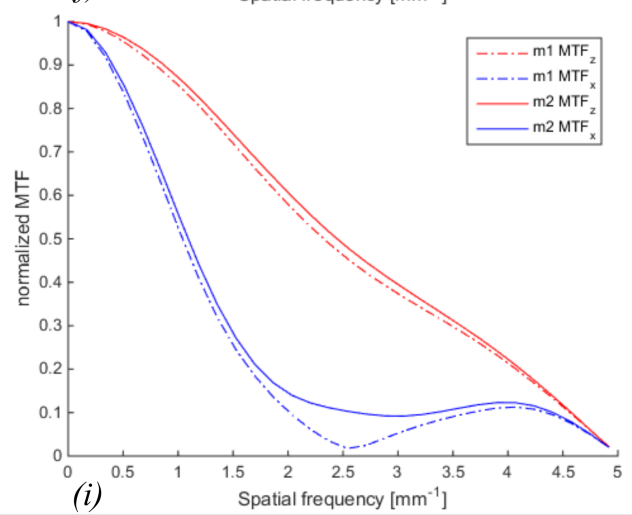

(i)

Figure 4: Results from the simulation study. The $M S E_{\text {proj }}$ and the $M S E_{\text {recon }}$ as well as all the SSIM components from the reconstructions using proposed Algorithm 1 with zero prior, defective boundary and perfect boundary respectively are plotted in $(\mathrm{a}, \mathrm{d}, \mathrm{g})$. All traces share the same horizontal axis which indicates the number of iterations, while the MSEs are plotted against the primary vertical axis on the left and the SIMM and its components apart from the structural component are plotted against the secondary vertical axis. The insets show the structural components of the SSIM. The cross section images of the reconstructed volume taken from the trajectory plane are shown in $(b, e, h)$. The gray level map window is $[-0.01,0.06]$. The two features used for detailed analysis are indicated in (b) as $f 1$ and $f 2$. The MTF measured along horizontal direction and vertical direction at $f 1$ and $f 2$ are illustrated in $(\mathrm{c}, \mathrm{f}, \mathrm{i})$. 


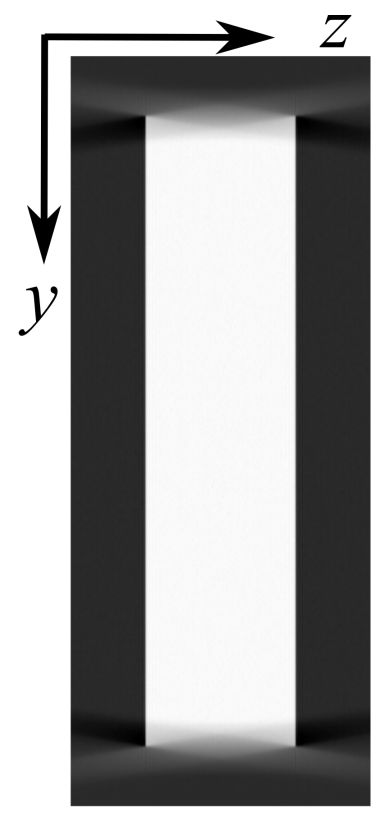

(a)

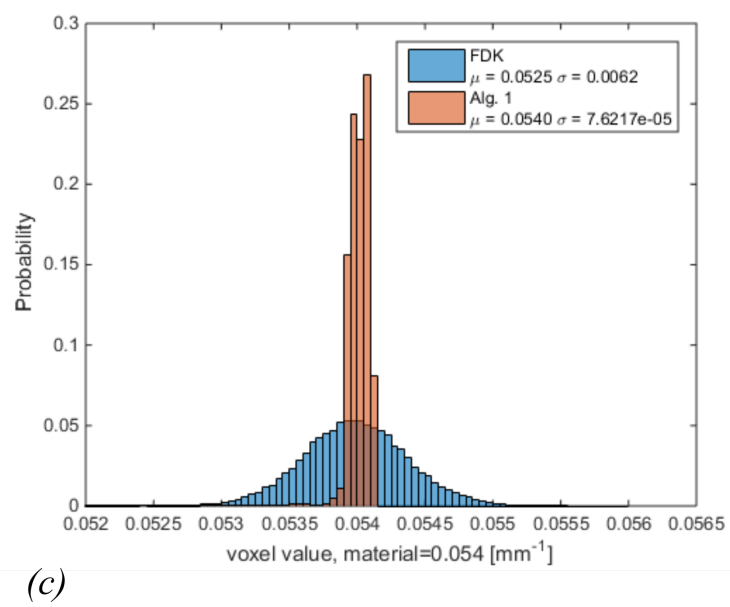

Figure 5: Comparasion of cone beam artifact between FDK and the proposed algorithm. The cross section from the $y-z$ plane from the FDK reconstruction is shown in (a), while the same cross section from the reconstruction with surface data is shown in (b). The histogram (normalized with probability) of all the pixel in the object for both cross section images is shown in (c). The gray value distributions are fitted against Gaussian distributions and the results are indicated in the legend of (c). 

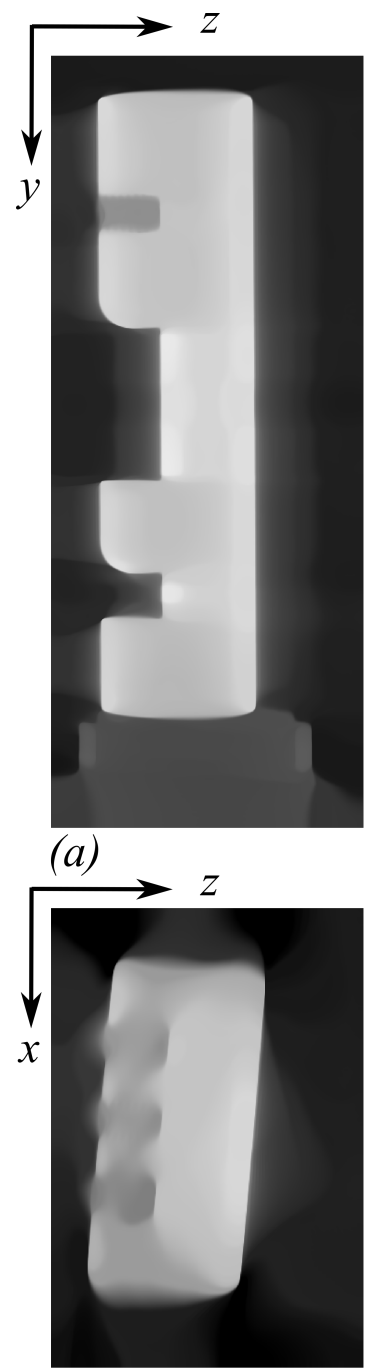

(c)
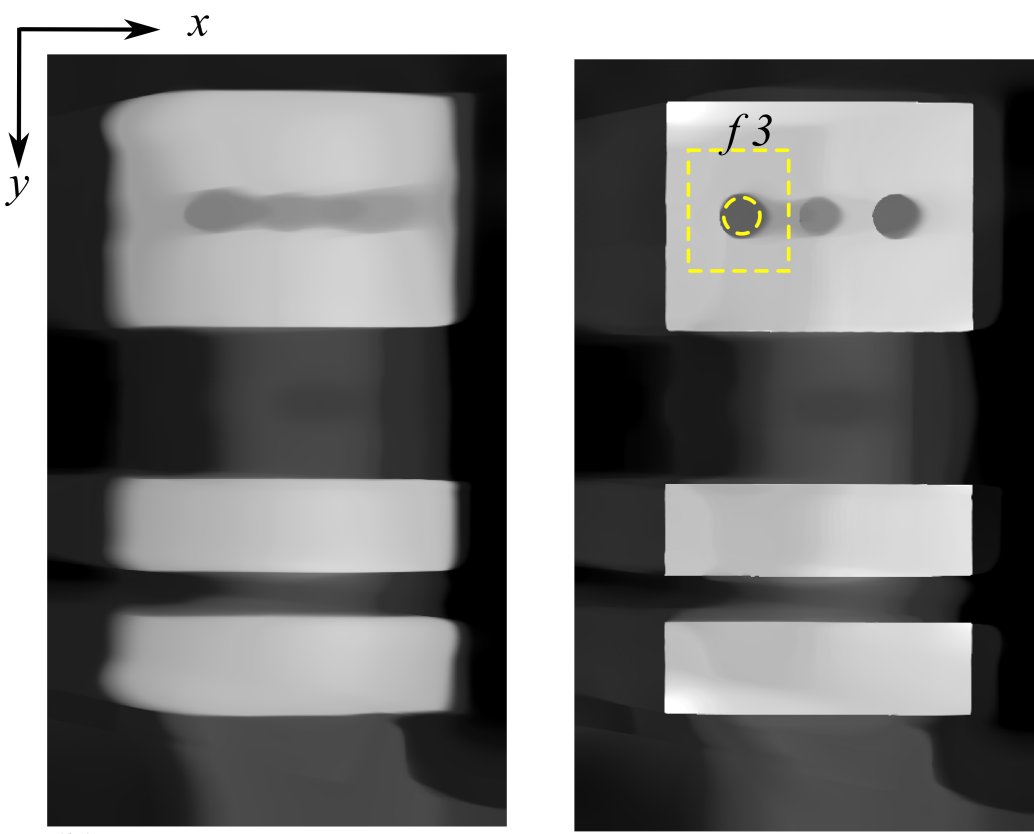

(b)

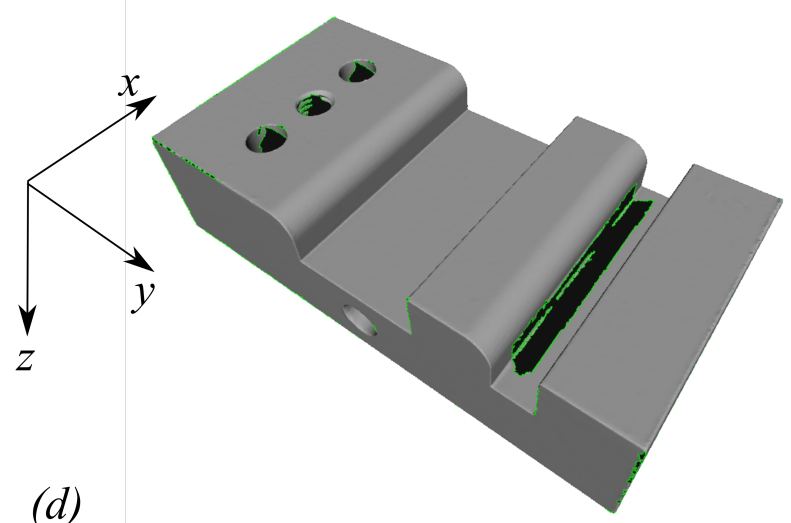

Figure 6: The cross sections from $y-z, x-y$ and $x-z$ planes are shown in pairs in (a)(b)(c). The reconstruction without optical scan, i.e. zero prior, is always placed on the left while the reconstruction with optical data, i.e. defective boundary, on the right. The features ( $f 3$ and $f 4)$ are highlighted in (b) and (c). The gray level window is $[-0.01,0.09]$. The mesh from the optical scan is rendered in (d). Due to the limitations of optical scan, the structures in the holes and part of the trench are not resolved and shown as holes in (d). 